\title{
Review of: "Skin-electrode iontronic interface for mechanosensing"
}

Wei $\operatorname{Lin}^{1}$

1 State University of New York at Stony Brook

Potential competing interests: The author(s) declared that no potential competing interests exist.

The paper presented a design of pressure sensor with high pressure sensitivity and spatial resolution. The design was validated through a series of experiments including the relative change of the capacitance to the applied pressure, the ability to sensor blood pressure pulses at the finger tip and the performance of the sensor during exercise and sweating. The sensor will have a broad applications in robotics and biomedical engineering.

The major concern of the study is that the sensor does not show the capability of quantitative measurement. It will be significant if the sensor can directly output the applied pressure value. This involves the study of the repeatability and the calibration of the sensor. It can a new study.

The minor concern is the cluster of figures. It would be helpful to the readers by breaking up the subfigures in a figure and place them close to the related text.

The figure number is missing in the first sentence of the second paragraph in first column on page 4. 